\title{
Camouflaged Foreground Detection with Fusion Methods
}

\author{
Muzammil Parvez $M^{1 *}$, K Suresh Kumar ${ }^{1}$, Sreevardhan Cheerla ${ }^{1}$, V. Subba Reddy ${ }^{1}$, Syed Inthiyaz $^{1}$, \\ Chennamsetty Pulla Rao ${ }^{2}$ \\ ${ }^{1}$ Department of Electronics and Communication Engineering, Koneru Lakshmaiah Education Foundation, \\ Vaddeswaram, AP, India \\ ${ }^{2}$ DVR \& Dr HS MIC College OF Technology, Kanchikacherla, Andhra Pradesh 521180
}

\begin{abstract}
Camouflage, also called as cryptic coloration, is a defense mechanism or tactic that organisms use to disguise their appearance, usually to blend in with their surroundings. Camouflage is used to mask their location, identity and movement. Detecting camouflaged moving foreground objects has been known to be difficult due to the similarity between the foreground objects and the background. Methods present to detect camouflage image i.e., decamouflaged techniques are i. Visual camouflaged breaking, ii. Co-occurrence matrix and invariant central moments, iii. Color and intensity based camouflaged detection, iv. Use of GLCM (gray level co-occurrence matrix) and dendrogram in camouflage detection, v. Color, edge and intensity-based background subtraction, vi. HSV (hue, saturation, value) color and GLCM texture to identify camouflaged object, vii.3D convexity-based camouflage detection method. In this paper we present a fusion framework to address this problem in wavelet domain. The proposed fusion framework is denoted by "FWFC" (fusion in the wavelet domain for foreground detection in camouflaged senses). We first show that the small differences in the image domain can be highlighted in certain wavelet bands. Then each wavelet coefficient of foreground is estimated by formulating foreground and background models for each wavelet band. The proposed fusion framework works on/from different wavelet bands based on the characteristics of the wavelet transform. In the field of military security and medical field (e.g.: drugresistant tuberculosis) this fusion framework can have many applications. This method has shown the significant performance which is better than the existing methods in terms of the camouflaged foreground detection.
\end{abstract}

Keywords: Maintenance of background, Texture, Prior and Posterior and Classifiers (Bayes).

\section{INTRODUCTION}

The techniques which transmogrify the world into an digital world like computer imaginative and machine intelligence works on process like taking amalgamation of data which is image and amplify them if required. These mimic the human behavior and decision-making power [1][2]. Such wise structures located their direction to many programs like smart phones, automated cars, surveillance systems etc; these technologies are the replica of human eyes and brain in lots of packages. The persisting assignment is to satisfy sensible sensitivity, fidelity, and accuracy constraints. Many fundamental areas of virtual imaging, computer vision and machine learning algorithm have up to date using the above improve generation. Digital cameras now have the aptitude of recording in high Definition (HD), high dynamic range (HDR) format [3], imitating the human visible system (HVS) near to perfection [4]. In a clever system an excellent best picture is captured it needs to be operated to reinforce excellent and to induce obviate any artifact so one can be created by the support of the gadgets those are captured [5]. Those are completed via the usage of picture processing algorithms like color correction, distortion elimination, polishing, deblurring etc..., in next level while pictures use computer imaginative and prescient algorithms like movement detection [6], foreground object extraction, item detection and so on..., to stumble on item of significance inside the scene environment. As soon as computers are detected, spotting their type a behavior requires machine algorithms [7]. The approach of this paper goes by capturing the video, preprocessing the data obtained [8], features extraction, background subtraction and foreground detection

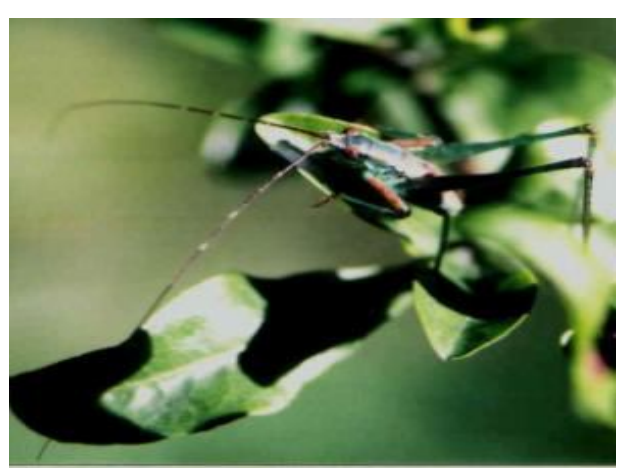



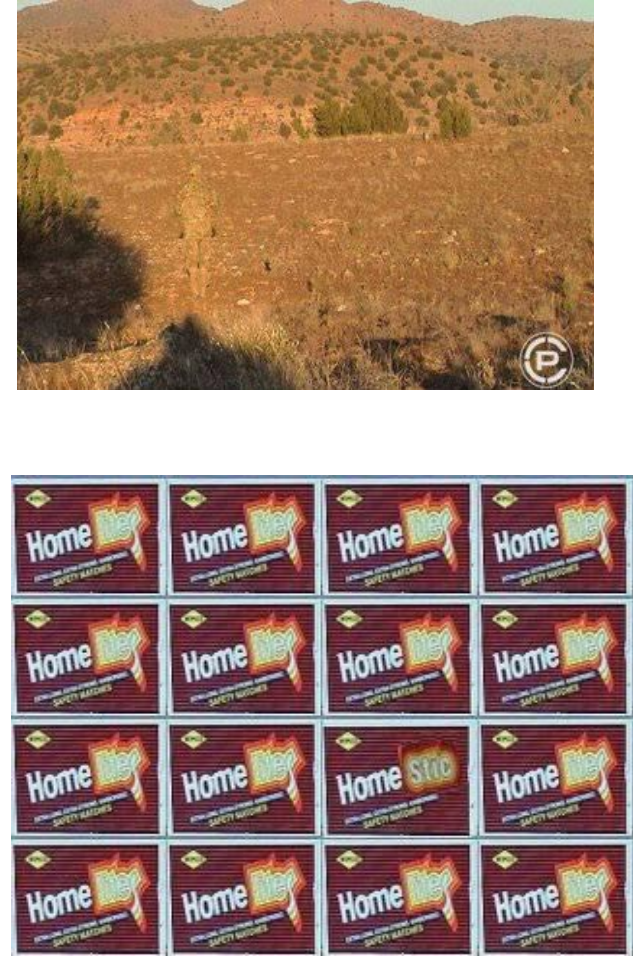

Figure 1: Example Camouflage insect, a man in the desert and Mixing up the duplicate
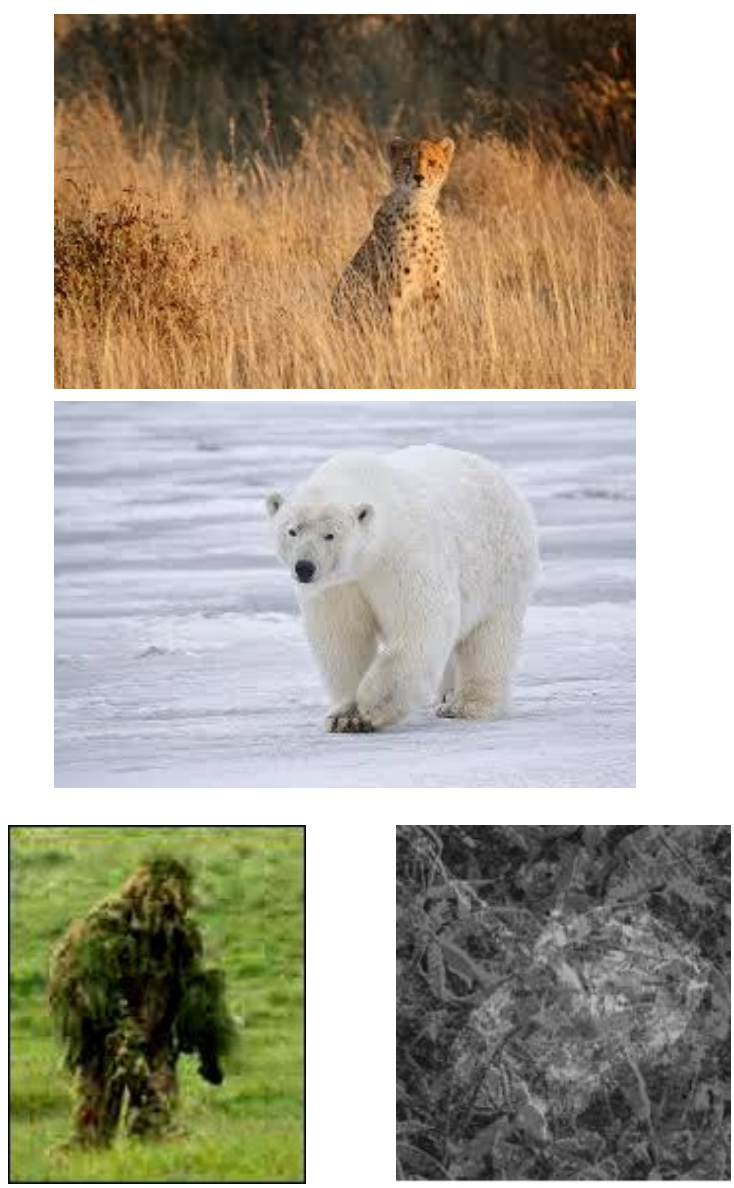

Figure 2: Camouflage leopard, Polar bear, a soldier in grass and military tank from the survey

\subsection{Final Stage}

The approach of workflow, human and animals to get similarities or look like the surroundings in the background [9][10]. The image which has this type of characteristics is called camouflaged image. These images are the combined images of foreground and background. In this image foreground object is hidden with the background [11][12]. Camouflaged images are of two types,
a) Natural Camouflage
b) Artificial Camouflage

\section{a) Natural Camouflage}

Natural camouflage exists within the nature which mostly performed by the ecosystem and natural environment without any human interaction [13][14]. In the nature, some carnivorous animals and plants adopt to the surrounding hide themselves to hunt their food. The animals change their colour and shape as required to the surrounding such that it is difficult to other animals or the predators to find them [15][16].

\section{b) Artificial Camouflage}

Artificial camouflage is a man-made camouflaged pattern. In military, the soldiers hide with respect to the surroundings and environment there by not possible to recognize by enemies. They mix up with the background as their texture gets involved in the surrounding

\section{PROPOSED METHOD}

The synthetic image is first decomposed into 4 types of wavelet bands [17][18]. They are LL band indicates approximation (A), LH band indicates horizontal $(\mathrm{H}), \mathrm{HL}$ band indicates vertical $(\mathrm{V})$ and $\mathrm{HH}$ band indicates diagonal (D). The formation of bands is done by transient a pixel to a high pass filter and low pass filter of an image. The above methodology is called stationary wavelet transform. The decision is taken in 0 's and 1 's, if the decision is ' 1 ' then the probability of finding a pixel in foreground is greater than probability of finding a pixel in background. If the decision is ' 0 ' then the pixel is in background.

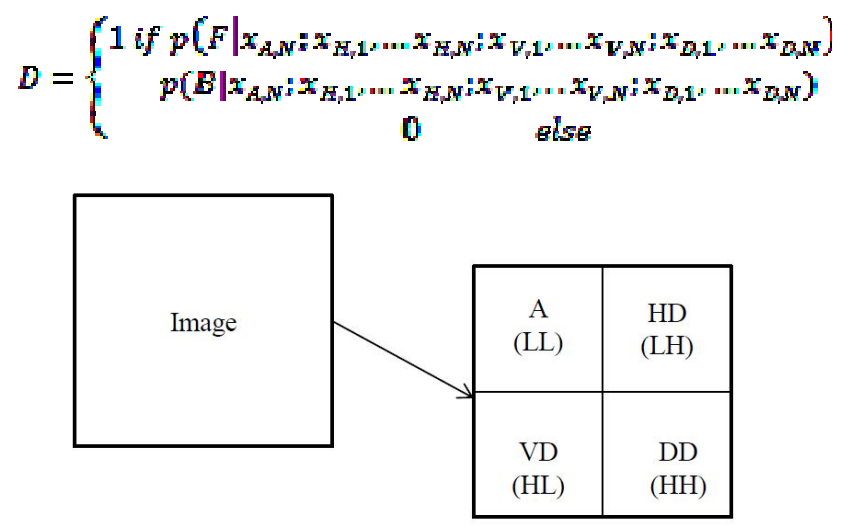

Figure 3: Conversion of image into wavelet bands 
By using Bayesian theorem, we can rewrite the equation as,

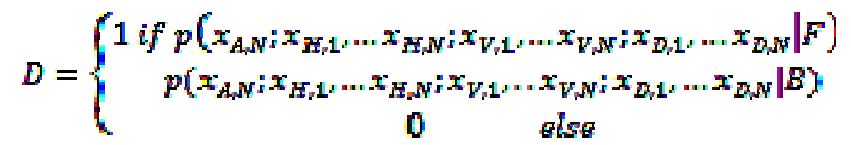

Foreground in an image typically occupies less than $50 \%$ of the image. So, for simplicity we assume $p(F)=p(B)$. Four wavelet bands are independent from each other as they capture different characteristics.

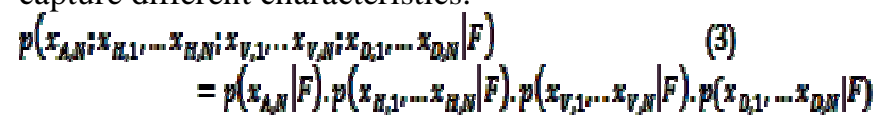

Level fusion is the process of combining the values of $\mathrm{N}$ levels of each band and getting the most probable value for each band. Similarly, band fusion fuses the results of different bands to obtain the result of foreground decision. A widely used process called Gaussian mixture models are used to formulate an image with Gaussian distributions.

$$
p\left(x_{d, \lambda}\right)=\sum_{m=1}^{M} \pi_{m} N\left(x_{d, l} \mu_{m} \delta_{\mathrm{m}}^{2}\right)
$$

Where $\mathrm{d}$ is wavelet band type and 1 is band level. Gaussian components are which indicates weight, mean and variance respectively.

The samples taken might be either background or foreground of an object. Foreground sample is taken as a small weight when compared with background sample as it has largest weight. In natural images foreground objects follow uniform distribution. The filter operation performed for coefficients of wavelets is taken as linear transformation, and then the Gaussian distribution is given as:

$$
p\left(x_{d, l} \mid F\right)=N\left(x_{d, l} F \mu_{F}, \delta_{F}^{2}\right)
$$

Where and are the mean and variance for the wavelet band $\mathrm{d}$ at level 1 of the foreground distribution.

$$
\begin{aligned}
& p\left(x_{d} \mid F\right)=p\left(x_{d, 1} \mid F\right) \cdot p\left(x_{d, 2} \mid F\right) \ldots p\left(x_{d, N} \mid F\right) \\
& p\left(x_{d} \mid B\right)=p\left(x_{d, 1} \mid B\right) \cdot p\left(x_{d, 2} \mid B\right) \ldots p\left(x_{d, N} \mid B\right)
\end{aligned}
$$

Wavelet bands obtain the results in the same line of direction for different levels those are related to each other. Anyway, the above decomposition doesn't perform well. So, we introduce the weighted fusion so that we can obtain functions of likelihood of each wavelet bands. Here the pixel blocks are formed so, that if the block size increases then level also increases. By reducing noise, the new functions of likelihood are: [19-21]

$$
\begin{aligned}
& p\left(x_{d} \mid F\right)=\frac{1}{N} \sum_{l=1}^{N} f\left(p\left(x_{d, l} \mid F\right)\right) \\
& p\left(x_{d} \mid B\right)=\frac{1}{N} \sum_{i=1}^{N} f\left(p\left(x_{d, l} \mid B\right)\right)
\end{aligned}
$$

Where $f($.$) maps the result from wavelet domain to image$ domain, which is a linear processing

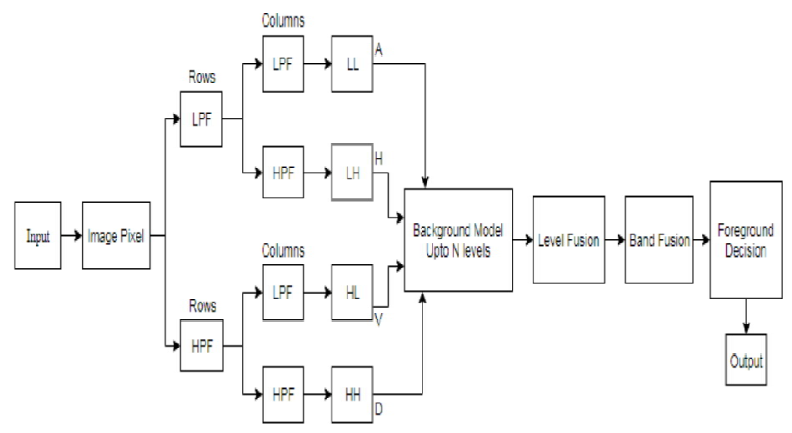

.Figure 4: Block diagram of the proposed framework

Sometimes the coefficients obtained may not satisfy for the object because the relationship between the foreground and background is nearly same. So, to characterize the pixels we take the correlation function so that we can correlate the pixels among them. Take first order auto regressive process.

$$
x(i)=c+\alpha \cdot x(i-1)+\varepsilon
$$

Where, the autoregressive coefficient lies between 0 and 1 . Finally, the correlation among pixels is given by:

$$
p(\delta)=a^{\delta}
$$

If the distances between two pixels increase then the correlation value reduces. Correlation values among pixels are different in different levels of bands. The pixel distance also changes for the center pixel to the far most pixel.

A translation weight is added, which determine the average correlation of all the pixels.

$$
\omega_{z, 1}=\frac{1}{N_{p}} \sum_{i=1}^{N_{p}} \rho\left(\delta_{i}\right)
$$

Where, the total number of pixels at level 1 and 0 is distance between pixel and center of the block.

In every image there exists some noise which disturbs our result. So, let us assume some noise with zero mean value i.e. white Gaussian noise which is different at different levels and bands. 


$$
\omega_{n, d, l}=\frac{\sigma_{d, l}-\sigma_{n}}{\sigma_{d, l}}
$$

Where ' $\sigma$ ' standard deviation of band is type $d$ at level 1 and is standard deviation of nose.

The final likelihood function for the wavelet bands is given as:

$$
\begin{aligned}
& p\left(x_{d \mid} \mid F\right)=\frac{1}{N} \sum_{i=1}^{N} \omega_{d, \lambda} p\left(x_{d, d} \mid F\right) \\
& p\left(x_{d} \mid B\right)=\frac{1}{N} \sum_{i=1}^{N} \omega_{d, \lambda} p\left(x_{d, d} \mid B\right)
\end{aligned}
$$

From the above equations the result is fused into a single band from different levels and then all the bands are further fused to obtain the decision of foreground detection.

\subsection{Bayes Classifier}

The Bayesian classifier is necessary to predict the values of features for members of given class. The main idea of Bayesian classifier is that if it knows the class, it can predict the values of other features. If it doesn't know the class, the Bayes rule is used to predict the class only if features of that class are known.

In Bayesian classifier, the learning agent builds a probabilistic model of the features. And that agent uses the developed model to predict the classification. Hence, Bayesian classifier is helpful for classification of foreground and background for us.

The classification theory of Bayes classifier uses a latent or hidden variable which is not observed. As mentioned above Bayesian classifier is a probabilistic model which yields a latent variable that is probabilistically related to the observed variables.

Bayesian classifier includes the conditional probability model in order to predict the future values depending up on the previously observed value. Both the predicted and observed values are conditionally independent of each other. This conditional probability is useful in classification in such a way that one can classify the particular value or feature belongs to. In the object detection scenario, it helps to classify that the pixel either belongs to foreground or background.[22-24]

Under Bayesian classifier, a theorem called as Bayes theorem is developed for probabilistic decision making. The posterior probability is obtained from the observed prior probability using Bayes theorem.

Let $\mathrm{A}$ and $\mathrm{B}$ are the two events then the Bayes theorem is mathematically represented as follows.

$$
P(A / B)=\frac{P(B / A) P(A)}{P(B)}(17)
$$

Where $\mathrm{P}(\mathrm{B} / \mathrm{A})$ the conditional probability of observing event $B$ given that $A$ is true.

Similarly, $\mathrm{P}(\mathrm{A} / \mathrm{B})$ is the probability of observing event $\mathrm{B}$ given that event $\mathrm{A}$ is true. $\mathrm{P}(\mathrm{A})$ and $\mathrm{P}(\mathrm{B})$ are probabilities of $\mathrm{A}$ and $\mathrm{B}$. Here, $\mathrm{A}$ and $\mathrm{B}$ are independent to each other.

Bayes classifier has wide range of applications in vision applications. It is used for classification of different objects and is also used in different algorithms like pattern classification, feature classification, pixel classification.

\subsection{Bayes Decision Rule}

Bayesian framework developed a decision theory for classification of background and foreground in modeling method ad it is Bayes decision theory. A formula is derived under Bayes decision rule for classification purpose.

In order to classify a region or scene let us consider a small position of the image pixel. Assume $\mathrm{s}=(\mathrm{x}, \mathrm{y})$ be the small position of an image pixel. Where $\mathrm{x}$ and $\mathrm{y}$ are the horizontal and vertical coordinates respectively. Let I ( $\mathrm{s}, \mathrm{t})$ be the input image at time $t$. ' $\mathrm{v}$ ' be the $\mathrm{n}$-dimensional feature vector obtained from the position $s$ and time $t$.

Then, from Bayes rule, posterior probability of ' $\mathrm{v}$ ' comes from the background at $\mathrm{s}$ is determined using formula.

$$
P_{s}(b / v)=\frac{P_{s}(v / b) P_{s}(b)}{P_{s}(v)}
$$

Here, prior probability is the original probability of outcome and the desired probability is obtained using posterior probability function.

Similarly, the posterior probability of feature vector ' $\mathrm{v}$ ' comes from the foreground at $\mathrm{s}$ is computed by,

$$
P_{s}(f / v)=\frac{P_{s}(v / f) P_{s}(f)}{P_{s}(v)}
$$

Using Bayes decision theory, pixel $\mathrm{s}$ at time $\mathrm{t}$ is classified as belonging to the background if the below condition is satisfied.

$$
P_{s}(v / b)>P_{s}(v / f)
$$

If the condition is not satisfied, then it can be concluded that the pixel s belongs to foreground.

The probability that the feature vector observed at an image pixel comes from either background or foreground is given as,

$$
P_{s}(v)=P_{a}(v / b) P_{a}(b)+P_{s}(v / f) P_{s}(f)
$$




\subsection{Seed-based region growing}

Seed-based region growing (SBRG) is the technique which performs the image segmentation by using a point which is called as seed. The selection of seed point is based upon user. The growth of a region starts from the seed point by combining the neighboring pixels having similar properties. A set of unallocated pixels which make borders of at least one of the regions is represented as

$$
P=\left\{x \notin \bigcup_{i=0}^{n} A_{i} \mid N(x) \cap \bigcup_{i=0}^{n} A_{i} \neq \phi\right\}
$$

If $\boldsymbol{i}(\boldsymbol{x}) \in\{1,2,3, \ldots$, is defined as the index such

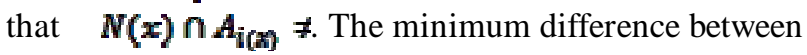
(x) and the region measure, is used as measure of similarity which is denoted as $\boldsymbol{\delta} \mathbb{k}$, where,

$$
\begin{gathered}
g(x)=\min \mid N(x)-\mu_{x \in A_{i}}[g(x)] \\
\mu(x)_{x \in A_{i}}=\frac{1}{n+1}\left[\sigma(x)+n^{*} \mu(x)_{x \in A_{i-1}}\right.
\end{gathered}
$$

\section{A. Seed Point Selection}

Number is automatic in nature. Analysis of spatial structures and shapes present in an image are assumed using morphology. Morphological operations like dilation, erosion, opening, closing and regional maxima are used for extracting, modifying and manipulating the features present in the image based on their structuring element. The shape and size of the structuring element plays an important role in image segmentation. Structuring of elements may be in different shapes like disk, square, line, rectangle, and rhombus etc using different morphological techniques.[25-27]

Mathematical representation of morphology of gray scale images considered as

$$
G(f)=\left\{(x, t) \in Z^{n} \times N_{0} \mid t=f(x)\right\}
$$

Local maxima are different from regional maxima where all the pixels of regional maxima are local maxima but converse is false. Dilation operator is used for representing local maxima so that a given pixel gives its maximum value compared with its neighbors given by structuring element B to its center.

\section{$\delta_{z}[G(f)]=\{(x, t) \mid B(x, t) \cap G(f) \neq \emptyset]$}

\section{SIMULATION}

When the wavelets are decomposed for all sub image locks, coefficients vector is gained. GLCM is calculated from the coefficients. Then wavelet coefficient features (WCF's) such as contrast, cluster shade and cluster prominence are found. Plotting is done by calculating WFC's for all the sub image blocks. Linear normalization is taken place for contrast and the rest two are logarithmic normalization. When a sub image has maximum sum of the features it can be considered as seed block.

Seed point is the center point of the seed block.Seed point is mainly useful in the region growing algorithm.

All the above-mentioned techniques are used in the present-day situations and problems and therefore further reducing the technical issues faced by those techniques our method can give good results and have high accuracy as well. The final conclusion is that the fusion frame work of conventional methods is very well managed and developed. The future work is the extension of this technique to be a fully automated recognition which can do using sensors and artificial intelligence so that a secured society can build up.

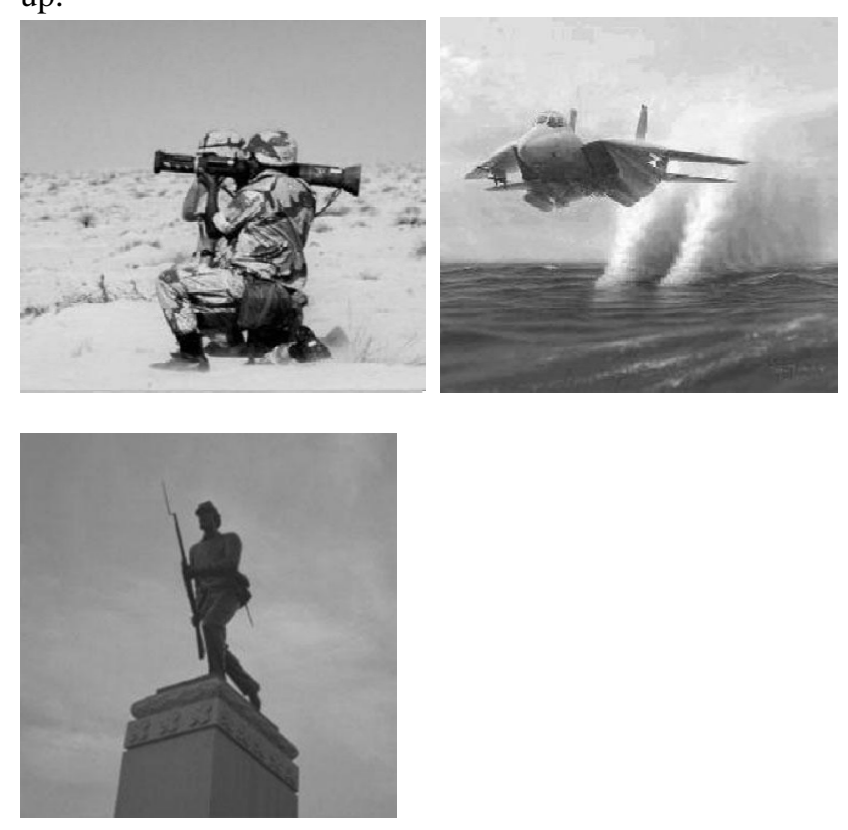

Figure 5: Samples images of Soldier, Aeroplane and Statue

\subsection{Target Extracted Image}

The sample images are extracted by detecting the foreground object
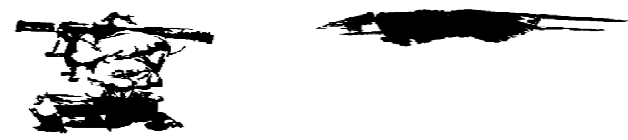


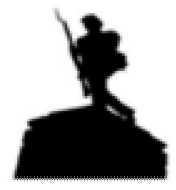

Figure 6: Target extracted images of Soldier, Aeroplane and Statue
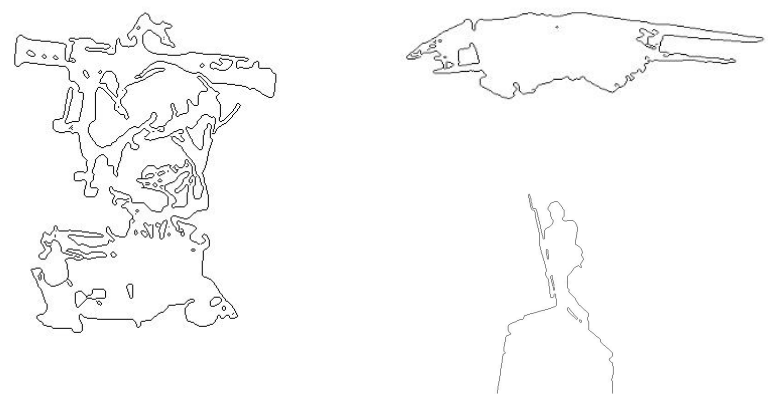

Figure 7: Canny edge detected images of Soldier, Aeroplane and Statue

\section{QUALITATIVE ANALYSIS}

After applying wavelet decomposition to all sub image blocks, we get coefficients vector. Gray level co-occurrence matrix is calculated from the coefficients.
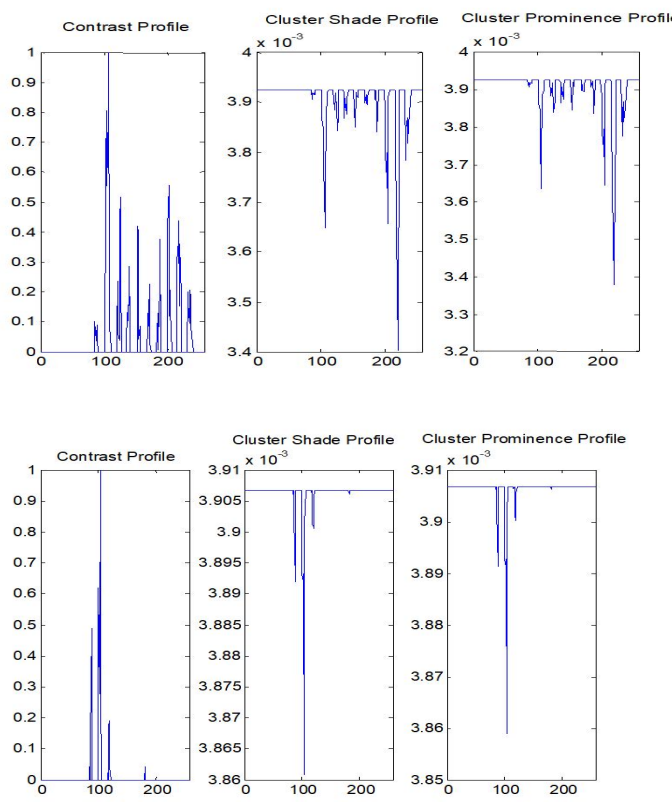

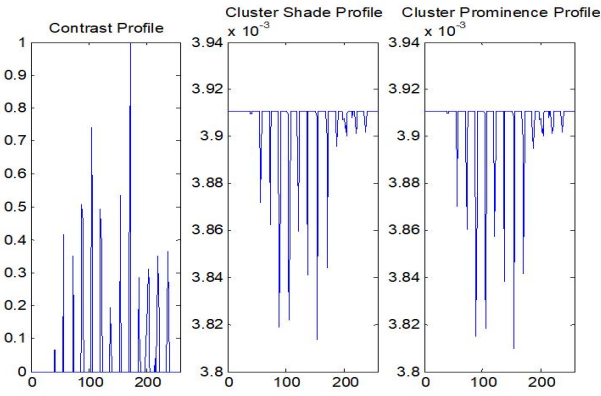

Figure 8: Profiles of wavelet coefficients features, Soldier, Aero plane and Statue respectively

Then wavelet coefficient features (WCF's) such as contrast, cluster shade and cluster prominence are found. Now WCF's are calculated for all the sub image blocks and they are plotted as above. Contrast is normalized linearly and the other two are normalized logarithmically. Sub image with maximum of the sum of the features [28-29] is treated as a seed block. Seed point is the center point of the seed block. Seed point is used in the region growing algorithm.

\section{CONCLUSION}

The all the above-mentioned techniques are used in the present-day situations and problems and therefore further reducing the technical issues faced by those techniques our method can give good results and have high accuracy as well. Comparative performance analysis is carried out using precision and recall for qualitative analysis. Precision and recall measures are better in statistical characterization with texture smoothing while comparing to without texture smoothing. In autonomous applications, illumination changes, shadows, time-varying background, light variation and random texture have to be addressed in the analysis of object detection. The final conclusion is that the fusion frame work of conventional methods is very well managed and developed. The future work is the extension of this technique to be a fully automated recognition which can do using sensors and artificial intelligence so that a secured society can build up.

\section{REFERENCES}

[1] S. Li, D. Florencio, W. Li, Y. Zhao, and C. Cook, "A Fusion Framework for Camouflaged Moving Foreground Detection in the Wavelet Domain," IEEE Trans. Image Process., vol. 27, no. 8, pp. 3918-3930, Aug. 2018, doi: 10.1109/TIP.2018.2828329.

[2] J. L. D. Shivani and R. K. Senapati, "Robust Image Embedded Watermarking Using DCT and Listless SPIHT," Futur. Internet 2017, Vol. 9, Page 33, vol. 9, no. 3, p. 33, Jul. 2017, doi: 10.3390/FI9030033.

[3] G. Suryanarayana and R. Dhuli, "Super-Resolution Image Reconstruction Using Dual-Mode Complex Diffusion-Based Shock Filter and Singular Value 
Decomposition," Circuits, Syst. Signal Process., vol. 36, no. 8, pp. 3409-3425, Aug. 2017, doi: 10.1007/s00034-016-0470-9.

[4] B. Bir bhanu, "Automatic Target Recognition: State of the Art Survey," IEEE Trans. Aerosp. Electron. Syst., vol. AES-22, no. 4, pp. 364-379, 1986, doi: 10.1109/TAES.1986.310772.

[5] K. V. V. Kumar, P. V. V. Kishore, and D. Anil Kumar, "Indian Classical Dance Classification with Adaboost Multiclass Classifier on Multifeature Fusion," Math. Probl. Eng., vol. 2017, 2017, doi: 10.1155/2017/6204742.

[6] "Gonzalez \& Woods, Digital Image Processing, 4th Edition | Pearson." https://www.pearson.com/us/higher-education/pro gram/Gonzalez-Digital-Image-Processing-4th-Edi tion/PGM241219.html (accessed Sep. 28, 2020).

[7] X. Zhang, C. Zhu, S. Wang, Y. Liu, and M. Ye, "A Bayesian Approach to Camouflaged Moving Object Detection," IEEE Trans. Circuits Syst. Video Technol., vol. 27, no. 9, pp. 2001-2013, Sep. 2017, doi: 10.1109/TCSVT.2016.2555719.

[8] C. Raghava Prasad and P. V. V. Kishore, "Performance of active contour models in train rolling stock part segmentation on high-speed video data," Cogent Eng., vol. 4, no. 1, p. 1279367 , Jan. 2017, doi: 10.1080/23311916.2017.1279367.

[9] T. Sirui, W. Chao, Z. Hong, Z. Bo, and W. Fan, "A wavelet based targets detection method for high resolution airborne SAR data," in International Geoscience and Remote Sensing Symposium (IGARSS), 2007, pp. 2071-2073, doi: 10.1109/IGARSS.2007.4423240.

[10] Sirisha B., Sandhya B., Paidimarry C.S., Sastry A.S.C. (2017),'Bag-of-Spatial Words(BoSW)Framework for Predicting SAR Image Registration in Real Time Applications',Procedia Computer Science, 115(),PP.431-439.

[11] Vallabhaneni, Ramesh Babu; Rajesh, V. "Brain tumour detection using mean shift clustering and GLCM features with edge adaptive total variation denoising technique" ALEXANDRIA ENGINEERING JOURNAL, Vol: 57, Issue no: 4 , pp: 2389 - 2392, 2018.

[12] S. Inthiyaz, B. T. P. Madhav, and P. V. V. Madhav, "Flower segmentation with level sets evolution controlled by colour, texture and shape features," Cogent Eng., vol. 4, no. 1, p. 1323572, Jan. 2017, doi: 10.1080/23311916.2017.1323572.

[13] C. S. Sastry, A. K. Pujari, B. L. Deekshatulu, and C. Bhagvati, "A wavelet based multiresolution algorithm for rotation invariant feature extraction," Pattern Recognit. Lett., vol. 25, no. 16, pp. 1845-1855, Dec. 2004, doi: 10.1016/j.patrec.2004.07.011.

[14] Aparna, Puvvadi; Kishore, Polurie Venkata Vijay.
"Biometric-based efficient medical image watermarking in E-healthcare application" IET IMAGE PROCESSING, Vol: 13 , Issue no: 3 , pp: $421-428,2019$.

[15] Soumya, I; Rahman, MZU; Reddy, DVRK; Lay-Ekuakille, A. "Efficient block processing of long duration biotelemetric brain data for health care monitoring" Review Of Scientific Instruments, DOI:10.1063/1.4913658.

[16] Siva D., Bojja P. (2019), 'Mlc based classification of satellite images for damage assessment index in disaster management', International Journal of Advanced Trends in Computer Science and Engineering, 8(6), PP.2825-283.

[17] Sampath Dakshina Murthy A., Satyanarayana Murthy P., Rajesh V., Hasane Ahammad S., Omkar Lakshmi Jagan B. (2019), 'Execution of natural random forest machine learning techniques on multi spectral image compression', International Journal of Pharmaceutical Research, 11(4), PP.1241-125.

[18] Pasha M.M., Subba Rao G.V., Suresh B., Tabassum S. (2019), 'Inspection of defects in CFRP based on principal components', International Journal of Recent Technology and Engineering, 8(3), PP.2367-2370.

[19] Bhavana D., Kishore Kumar K., Rajesh V., Swetha Sree M., Mounika D., Bhavana N. (2019), 'Deep learning for pixel-level image fusion using CSR technique', International Journal of Recent Technology and Engineering, 8(2), PP.792-797.

[20] Pardhasaradhi P., Madhav B.T.P., Sindhuja G.L., Sreeram K.S., Parvathi M., Lokesh B. ( 2018), 'Image enhancement with contrast coefficients using wavelet based image fusion',International Journal of Engineering and Technology(UAE), 7 (2.8 Special Issue 8),PP. 432- 435.

[21] Tripathi D.P., Pardhasaradhi P., Madhav B.T.P. ( 2018) , 'Statistical parameters-based image enhancement techniques in pure and nanodispersed 60.08 liquid crystalline compounds',Phase Transitions, 91 (8),PP. 821832.

[22] Kanjalkar P.M., Patil P.T., Rao M.V.G. ( 2018), 'Image reconstruction based on combination of modified CoSaMP and orthogonal wavelets',2017 International Conference on Intelligent Computing, Instrumentation and Control Technologies, ICICICT 2017, 0 (),PP. 1401- 1408

[23] Vijaya Lakshmi, A., Ghali, V.S., Muzammil Parvez, M., Chandra Sekhar Yadav, G.V.P., Gopi Tilak, V. "Fuzzy C-means clustering based anomalies detection in quadratic frequency modulated thermal wave imaging"International Journal of Recent Technology and Engineering Volume-8 Issue-3, September 2019

[24] Network security using notable cryptographic 
algorithm for IoT data Muzammil Parvez, M., Ravindran, R.S.E., Inthiyaz, S., Tejkumar, C., Veera Ram Sai, K., Shiva Reddy, K.A. International Journal of Emerging Trends in Engineering Research Volume-8 Issue-5 May 2020

[25] Alive node and network lifetime analysis of DEEC protocol and EDDEEC protocol Muzammil Parvez, M., Shanmugam, J., Mohan Rao, K.R.R., Lakshmana, C., Shameem, S. Journal of Advanced Research in Dynamical and Control Systems Volume10( Special Issue-6),2018

[26] Machine learning based automatic defect detection in non stationary thermal wave imagingVijayalakshmi,A.,Ghali,V.S.,Chandrasek har Yadav, G.V.P., Gopitilak, V., Muzammil Parvez,M, ARPNJournalof Engineering and Applied Sciences Volume15 Issue 2,2019

[27] Decision tree-based subsurface analysis using Barker coded thermal wave imaging Parvez M, M., Shanmugam, J., Ghali, V.S. Infrared Physics and Technology109,2020.

[28] Sureshkumar K., Chandra Vamsi G., Chandu L., Nikhil B., Chetan Sai K. ( 2018), 'Implementation of meander line based reconfigurable CPW-fed antenna',Journal of Advanced Research in Dynamical and Control Systems, 10 (2),PP. 579583

[29] Inthiyaz S, Madhav B, Kishore P, " Flower image segmentation with PCA fused colored covariance and gabor texture features based level sets", Ain Shams Engineering Journal (2018) 9(4) 3277-3291. 